International Journal of Modern Physics A

(C) World Scientific Publishing Company

\title{
EMERGENT SPACETIME AND THE COSMOLOGICAL CONSTANT
}

\author{
HYUN SEOK YANG \\ School of Physics, Korea Institute for Advanced Study, Seoul 130-012, Korea \\ hsyang@kias.re.kr
}

Received 25 March 2008

\begin{abstract}
We address issues on the origin of gravity and the dark energy (or the cosmological constant) from the perspectives of emergent gravity. We discuss how the emergent gravity reveals a noble, radically different picture about the origin of spacetime, which is crucial for a tenable solution of the cosmological constant problem. In particular, the emergent gravity naturally explains the dynamical origin of flat spacetime, which is absent in Einstein gravity.

Keywords: Emergent Spacetime; Cosmological Constant; Noncommutative Field Theory

PACS numbers: 11.10.Nx, 98.80.Cq, 04.50.Kd
\end{abstract}

\section{The Cosmological Constant and Emergent Spacetime}

We suggested in Ref. 1 that the cosmological constant (CC) problem can be resolved in a natural way if gravity emerges from a gauge theory in noncommutative (NC) spacetime. Especially, it was shown that the emergent gravity formulated from a background independent theory reveals a noble, radically different picture about the origin of spacetime where vacuum energy does not gravitate but only fluctuations around the vacuum generate gravity. An extreme example is that a flat spacetime emerges from uniform condensation of energy, previously identified with the CC.

In this short contribution we will summarize an underlying spacetime picture emerging from the background independent formulation of NC gauge theory. Some technical details will be deferred to Refs. 2 and 3 .

In general relativity, gravity is described by metric fields of spacetime which is determined by the distribution of matter and energy. That is, the spacetime geometry is not a rigid but a dynamical object. But there is a blind point about the dynamical origin of spacetime in general relativity; it says nothing about the dynamical origin of flat spacetime since the flat spacetime is a geometry of special relativity rather than general relativity. It turns out 1 that this nonchalance about the dynamical origin of flat spacetime gives rise to the $\mathrm{CC}$ problem in general relativity.

However, it has been shown in Refs. 2,3 that the emergent gravity from NC gauge theory naturally explains the dynamical origin of flat spacetime, which is absent in Einstein gravity. We want to emphasize that this spacetime picture is an inevitable consequence provided one accepts the fact that gravity is an emergent phenomenon, 
which nowadays becomes a new paradigm emerging from string theory.

If gravity is emergent from gauge fields, the general relativity implies that spacetime should also be emergent from gauge field interactions a. If so, what kind of gauge field configurations corresponds to a flat spacetime ? The most tenable answer is a uniform condensation of gauge fields in a vacuum, while it appears as a vacuum energy in Einstein gravity, so causing the CC problem. Therefore "a solution" of the CC problem might be surprisingly simple if and only if there exists a physically viable theory (i.e., a background independent theory) to contain all the above properties. The correspondence between $\mathrm{NC}$ field theory and gravity $2 \mid 3$ precisely realizes the desired properties of emergent gravity and so resolves the CC problem 1 .

A NC spacetime arises from a condensation of gauge fields in a vacuum:

$$
\left\langle B_{a b}\right\rangle_{\mathrm{vac}}=\left(\theta^{-1}\right)_{a b} \Leftrightarrow\left[y^{a}, y^{b}\right]_{\star}=i \theta^{a b} \Leftrightarrow\left[a_{i}, a_{j}^{\dagger}\right]=\delta_{i j}
$$

where $a, b=1, \ldots, 2 n$ and $i, j=1, \cdots, n$. Every $\mathrm{NC}$ space can be represented as a theory of operators in a Hilbert space $\mathcal{H}$, which consists of (NC) $\mathrm{C}^{*}$-algebra $\mathcal{A}_{\theta}$ like as a set of observables in quantum mechanics. A standard dynamical system can be described in terms of vector fields as derivations of a certain (commutative) $\mathrm{C}^{*}$-algebra. This concept of dynamics can be generalized to a $\mathrm{NC}$ space provided that one describes $\mathrm{NC}$ dynamics in terms of derivations of $\mathrm{NC} \mathrm{C}^{*}$-algebra. This kind of vector fields in the $\mathrm{NC}$ space (11) was explicitly constructed in Ref. 2, where it was shown that the vector fields form an orthonormal frame and so define vielbeins of a gravitational metric.

Furthermore, if one has a representation of the Hilbert space $\mathcal{H}$, e.g., a Fock space of harmonic oscillators for the space (1), any operator in $\mathcal{A}_{\theta}$ or any NC field can be represented as a matrix whose size is determined by the dimension of $\mathcal{H}$. For the NC space (11), one gets $N \times N$ matrices in the $N \rightarrow \infty$ limit. In this sense, the emergent geometry arising from the vector fields in the NC space (11) can be understood as a dual geometry of large $N$ matrices in $\mathcal{H}$ according to the large $N$ duality or AdS/CFT correspondence $2[3$.

A looming point in emergent gravity is that a flat spacetime emerges from the vacuum (11) triggered by the Planck energy condensation. In other words, the vacuum

energy does not gravitate and so causes no CC problem 1. A flat spacetime is not free gratis but a result of the maximum energy condensation in a vacuum.

\section{References}

1. H. S. Yang, Emergent Gravity And The Cosmological Constant Problem, arXiv:0711.2797.

2. H. S. Yang, Instantons and Emergent Geometry, hep-th/0608013 Emergent Gravity from Noncommutative Spacetime, hep-th/0611174 Noncommutative Electromagnetism As A Large N Gauge Theory, arXiv:0704.0929.

3. H. S. Yang, Mod. Phys. Lett. A22, 1119 (2007); Noncommutative Spacetime and Emergent Gravity, arXiv:0711.0234

\footnotetext{
${ }^{a}$ Here we suppose that there exists a background independent theory where any spacetime structure is not a priori assumed but defined from the theory.
} 